\title{
Perfusion-Weighted Imaging-Derived Collateral Flow Index is a Predictor of MCA M1 Recanalization after IV Thrombolysis
}

\author{
F. Nicoli, P. Lafaye de Micheaux, and N. Girard
}

\begin{abstract}
BACKGROUND AND PURPOSE: Recent studies highlight the role of CC in preserving ischemic penumbra. Some authors suggested the quality of CC could also impact recanalization. The purpose of this study is to test this hypothesis in patients who were treated with IV thrombolysis for MCA-M1 occlusion.
\end{abstract}

MATERIALS AND METHODS: A normalized index derived from Tmax maps (MR-PWI) was defined to quantify the CC deficit ( $\mathrm{nCCD}$ ) in 64 patients with stroke who underwent IV thrombolysis. Correlations between nCCD and parameters that may be altered by CC quality were tested (baseline NIHSS, volume of diffusion abnormalities, modified Rankin Scale at 3 months). The correlation between baseline nCCD and MCA-M1 recanalization rate at 24 hours was tested.

RESULTS: The nCCD is significantly correlated with NIHSS and with lesional volume (Pearson correlation test, positive correlations, respectively, $0.40,0.57 ; P=.00089, P=8.7 \mathrm{e}-07)$. The $\mathrm{nCCD}$ also has a significant predictive value on the full recanalization at 24 hours that decreases as TTT increases (logistic regression, $P=.021$ ). Furthermore, among patients who were treated within 3 hours, nCCD and recanalization are significantly correlated (correlation ratio test, eta2 $=0.23, P=.0023$ ): Patients who did not achieve full recanalization have significantly higher nCCD than fully recanalized patients (Mann-Whitney $U$ test, $P=.007$ ). In addition, the probability of full recanalization decreases as the nCCD increases $(P=.021)$. nCCD (OR 0.988, 95\% Cl 0.977-0.999, $P=.042$ ) and full recanalization at 24 hours (OR 4.539, 95\% Cl 1.252-16.456, $P=.021$ ) are independent predictors of functional independence at 3 months.

CONCLUSIONS: The nCCD index is a predictor of full MCA-M1 recanalization in patients treated with IV thrombolysis.

ABBREVIATIONS: $\mathrm{AIF}=$ arterial input function; $\mathrm{CC}=$ collateral circulation; $\mathrm{Cl}=$ confidence interval; $\mathrm{nCCD}=$ normalized collateral circulation deficit; Tmax $=$ time-to-maximum of the flow-scaled residue function; TTT = time-to-treatment (equivalent to onset-to-needle time)

A $\mathrm{n}$ increasing number of studies have recently highlighted the importance of CC in the final outcome after IV thrombolysis or intra-arterial thrombectomy in patients with acute stroke. ${ }^{1-7}$ All these studies focused on the role of CC in the preservation of the ischemic penumbra until recanalization is obtained. Some authors ${ }^{1}$ have suggested that the quality of CC could impact the recanalization rate, because the collateral flow may increase recanalization in MCA occlusion treated with IV thrombolysis by delivering the drug to the distal end of the clot. However, to our

Received February 9, 2012; accepted after revision April 2.

From the Service d'Urgences Neuro-Vasculaires (F.N.) and Service de Neuroradiologie (N.G.), Assistance Publique-Hôpitaux de Marseille (APHM), CHU de la Timone, Marseille, France; and Département de Mathématiques et Statistique (P.L.d.M.), Université de Montreal, Montreal, Canada.

Please address correspondence to Pr François Nicoli, Olea Medical, 93 ave.des sorbiers, ZI Athelia IV, F-13600 La Ciotat, France; e-mail: pr.f.nicoli@gmail.com

- Indicates open access to non-subscribers at www.ajnr.org

http://dx.doi.org/10.3174/ajnr.A3174 knowledge, no specifically designed study has aimed to validate this hypothesis in the literature.

The purpose of the current study is to test this hypothesis in patients with MCA-M1 occlusion treated with IV thrombolysis. To do so, a new appropriate index derived from MR perfusion imaging has been defined to indirectly quantify the CC deficit. Correlations between this newly defined parameter and other parameters that are supposed to be altered by CC deficit have been tested as well—for example, baseline NIHSS, volume of diffusion abnormalities, and the modified Rankin Scale at 3 months. Finally, the original hypothesis has been tested via a correlation study between the CC deficit index and the recanalization rate.

\section{MATERIALS AND METHODS Study Design}

This is a retrospective analysis of a subgroup of patients originally recorded in a prospective clinical registry for IV thrombolysis (FN) at La Timone Hospital, Marseille, France.

Age, sex, medical history, previous antithrombotic therapy, 
TTT, door-to-needle time, NIHSS, and modified Rankin Scale scores and imaging characteristics (presence of occlusion, thrombus location, results of initial MR imaging and follow-up CT and CTA) were consistently recorded in the registry and were therefore available for retrospective analysis.

The following are the patient selection criteria used in this study:

1) MR imaging performed before IV thrombolysis.

2) IV thrombolysis for MCA-M1 occlusion, using a standard dose of alteplase. $^{8}$

3) Satisfactory-quality PWI records available for analysis.

4) Control of MCA recanalization 24 hours after IV thrombolysis using CTA.

5) Clinical follow-up 3 months after stroke onset.

From January 2009 to December 2010, 262 patients were recorded in the registry. Sixty-four patients met the aforementioned selection criteria and were included in the current study.

Within the 3-hour time window, patients met standard inclusion and exclusion criteria. ${ }^{8}$ In the 3- to 4.5 -hour window, patients met European Cooperative Acute Stroke Study 3 inclusion and exclusion criteria. ${ }^{9}$ IV thrombolysis was exceptionally performed between 4.5 and 5.5 hours in 7 patients with fluctuating neurologic deficit and isolated MCA-M1 occlusion $(71.4 \%$ distal). The decision to perform IV thrombolysis in these patients, meeting other European Cooperative Acute Stroke Study 3 inclusion and exclusion criteria, was based on very strict criteria, including normal brain parenchyma on $\mathrm{T}^{*}$ and FLAIR images, preserved CBV inside ischemic area, DWI lesions volume $<20 \mathrm{~mL}$, obvious diffusionperfusion mismatch, and informed consent from relatives.

In our hospital, all patients with acute ischemic stroke who are potentially eligible for IV thrombolysis have 24/7 priority access to MR imaging (French national recommendations for early stroke management, http://www.has-sante.fr/portail/jcms/c_830203). Upon arrival at the hospital, they are directly transferred to the MR imaging department to a dedicated room equipped with vital parameters monitoring systems, located close to MR scanners. Once blood samples are taken, MR imaging is performed within 10 minutes. In our practice, DWI and PWI postprocessing does not exceed 3 minutes. IV thrombolysis is performed immediately after the MR examination in a dedicated room of the MR department. In the department, mean door-to-needle time is 35 minutes. ${ }^{10}$

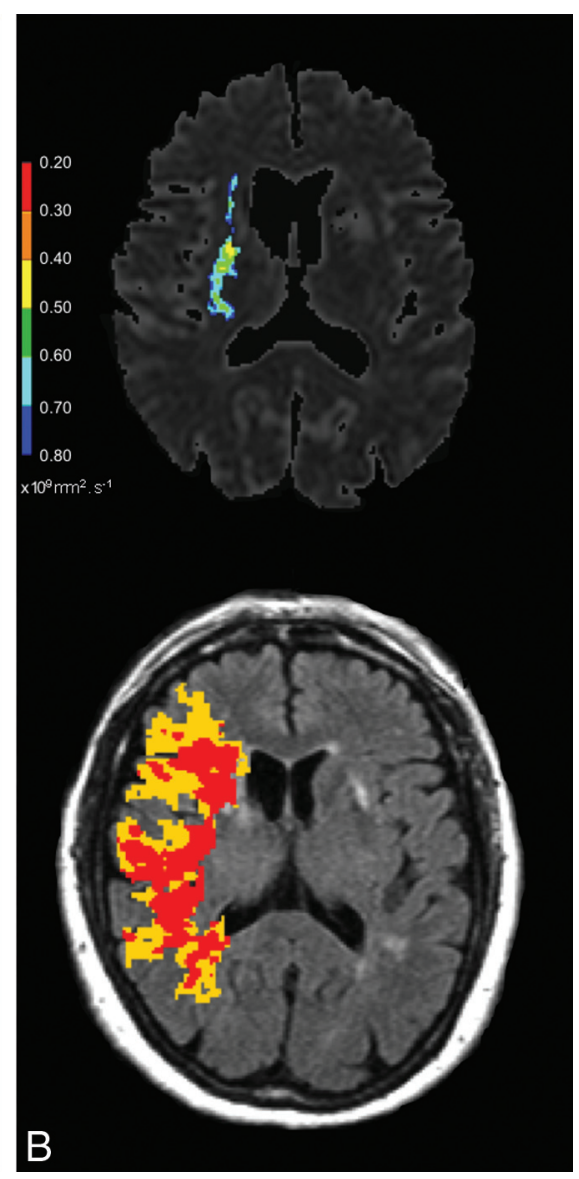

FIG 1. Tmax maps used for $n C C D$ quantification and corresponding ADC maps from 2 patients with different recanalization pattern. Upper images: ADC maps with lesion delineated by visual selection of the most appropriate ADC threshold value (the hotter the color, the lower the ADC value). Lower images: Tmax maps (superimposed on FLAIR images) with colored areas corresponding to moderately (yellow area) and severely (red area) hypoperfused parenchyma. $A$, 75-year-old woman, proximal MCA-M1 occlusion, NIHSS $=11$, TTT = 135 minutes, no MCA recanalization at 24 hours. Volume of DWI abnormalities detected on all brain sections $=54.78$ $\mathrm{mL} . \mathrm{nCCD}=105.14$. B, 74-year-old woman, distal MCA-M1 occlusion, NIHSS $=15, \mathrm{TTT}=94$ minutes, complete MCA recanalization at 24 hours. Volume of DWI abnormalities detected on all brain sections $=2.74 \mathrm{~mL}$. $\mathrm{nCCD}=68.16$. Calculation method for $\mathrm{nCCD}$ : The yellow area corresponds to moderately hypoperfused parenchyma (Tmax values between lower and upper max thresholds). The red area corresponds to severely hypoperfused parenchyma (Tmax exceeding the upper threshold). Lower and upper thresholds were defined as the Tmax mean value of the whole contralateral hemisphere (calculated from a brain section located at the tralateral Tmax mean value +2 seconds and the upper threshold is contralateral Tmax mean value +6 seconds. The volume of ipsilateral and supratentorial, moderately (yellow area) or severely (red area), hypoperfused parenchyma is calculated from all brain sections. nCCD = (red area volume/yellow area volume) $\times$ (red area volume + yellow area volume).

As part of the routine protocol, systematic standard continuous 2-MHz transcranial Doppler monitoring was performed in all patients with MCA-M1 occlusion throughout the 2 hours following tPA intravenous bolus.

\section{Image Acquisition and Postprocessing}

MR examinations were performed on a 1.5T Intera (Philips Healthcare, Best, the Netherlands). All patients underwent DWI, FLAIR, T2* gradient-echo, and 3D time-of-flight MRA sequences. Diffusion was measured at 3 values $(b=0, b=500, b=$ 1000 seconds $/ \mathrm{mm}^{2}$ ). Average ADC maps were automatically generated. Axial dynamic gradient-echo echo-planar PWI was performed after tracking a bolus of $0.1 \mathrm{mmol} / \mathrm{kg}$ gadodiamide (Dota- 
Table 1: Reproducibility of collateral circulation deficit measurements: Interrater

\begin{tabular}{lccccc}
\hline & $\begin{array}{c}\text { Expert vs } \\
\text { Nonexpert 1 }\end{array}$ & $\begin{array}{c}\text { Expert vs } \\
\text { Nonexpert 2 }\end{array}$ & $\begin{array}{c}\text { Nonexpert 1 vs } \\
\text { Nonexpert 2 }\end{array}$ & ICC (95\% CI) \\
\hline Automatic AIF selection & $r^{2}$ & 0.84 & 0.90 & 0.94 & $0.92(0.88-0.96)$ \\
Manual AIF selection & $r^{2}$ & 0.77 & 0.79 & 0.84 & $0.88(0.82-0.92)$ \\
\hline
\end{tabular}

Note:-ICC indicates intraclass correlation.

Table 2: Reproducibility of collateral circulation deficit measurements: Intrarater (automatic vs manual AIF selection)

\begin{tabular}{lccc}
\hline & Expert & Nonexpert 1 & Nonexpert 2 \\
\hline$r^{2}$ & 0.72 & 0.86 & 0.85 \\
ICC $(95 \% \mathrm{CI})$ & $0.81(0.69-0.89)$ & $0.82(0.71-0.89)$ & $0.84(0.74-0.90)$ \\
\hline \multicolumn{2}{l}{ Note:-ICC indicates intraclass correlation. }
\end{tabular}

rem; Guerbet, Aulnay-sous-Bois, France). After an automatic selection of the AIF, maps of CBV, CBF, MTT, TTP, and Tmax were calculated and analyzed using Perfscape and Neuroscape software (Olea Medical, LA Ciotat, France). The volume of diffusion anomalies was quantified through an analysis of isotropic $b$-1000 DWI and ADC maps.

CT angiography was performed with a 64-detector Somatom Sensation CT scanner (Siemens Healthcare, Erlangen, Germany) to evaluate recanalization 24 hours after IV thrombolysis.

\section{Automatic Selection of the Arterial Input Function and Deconvolution Algorithm}

The automatic selection of the AIF is based on a clustering algorithm and classifies curves using the area under the curve, the roughness, and the first moment to discard arterial signal from tissular signal. ${ }^{11}$ The quantitative perfusion maps were obtained by using the singular value decomposition with a block-circulant deconvolution matrix algorithm. ${ }^{12}$

\section{Calculation of the Collateral Flow Index}

To achieve this goal, a new MR perfusion index derived from Tmax maps was calculated. This index of collateral circulation deficit is based on the ratio of volume of critically hypoperfused area (absolute Tmax value $>6$ seconds $^{13,14}$ in the current study) over the volume of moderately hypoperfused area (absolute Tmax value between 2 and 6 seconds in the current study), which was proposed by Bang et $\mathrm{al}^{6}$ for quantifying the severity of brain perfusion defects. Indeed, although these authors used a lower critical Tmax threshold (4 seconds), this ratio was significantly correlated with angiographic collateral grading in patients with MCA occlusion. ${ }^{6}$ To avoid artifact biases in volume computations, only ipsilateral and supratentorial areas were selected. The whole hypoperfused volume (volume of absolute Tmax value $\geq 2$ seconds) weighted the collateral circulation deficit index so as to avoid biases related to the site of arterial occlusion and to interindividual brain volume variability (Fig 1). Thus, collateral circulation deficit $=$ volume of absolute Tmax value $\geq 2$ seconds $\times$ (volume of absolute Tmax value $>6$ seconds/volume of absolute Tmax value between 2 and 6 seconds).

The new index defined in this study, named normalized collateral circulation deficit, or nCCD, is obtained by normalizing collateral circulation deficit with regard to the contralateral Tmax mean value (calculated from a brain section located at the level of the centrum semiovale, with exclusion of ventricles in each patient) to prevent interindividual AIF delay biases. Therefore, $\mathrm{nCCD}=$ collateral circulation deficit calculated with relative Tmax thresholds defined as contralateral Mean Tmax +2 seconds or $+6 \operatorname{seconds}^{13}$ (Fig 1 ).

\section{Reproducibility of Collateral Circulation Deficit Measurements}

The reproducibility of collateral circulation deficit measurements was assessed in a purposefully designed 3-rater study on 49 cases (subgroup of the current series). Three raters ( 1 neuroimaging expert and 2 nonexpert raters, fully blinded to clinical data) postprocessed all images twice, once with manual AIF selection and once with automatic AIF selection. Pearson correlation tests were used to establish intrarater automatic AIF versus manual AIF correlations as well as interrater correlations. Interclass coefficients were calculated to establish the level of intra- and interrater agreement. The results showed a nearly perfect reproducibility of collateral circulation deficit measurement, regardless of the rater's level of expertise or choice of AIF selection (Tables 1 and 2).

\section{Statistical Analysis}

An independent bio-statistician (P.L.d.M.) performed the statistical analysis. All statistical tests were performed by $\mathrm{R}^{\star}$ software, version 2.11.0 (http://www.r-project.org/). ${ }^{15}$

The univariate analysis consisted of

1) Student $t$ test or Mann-Whitney $U$ test for the comparison of mean values between 2 groups or ANOVA test for the comparison between 3 groups.

2) $\eta^{2}$ (eta2) correlation ratio test to measure the strength of the association between a quantitative and qualitative variable.

3) Pearson correlation tests to measure the strength of the association between 2 quantitative variables.

4) Fisher exact test for the comparison between percentages.

Logistic regressions were performed by the generalized linear model procedure ( $\mathrm{R}^{\star}$ software). ${ }^{15,16}$ The results were validated by using the Hosmer-Lemeshow test. A dispersion parameter equal to 1 was used within the generalized linear model procedure. Thus, the variance for the error term of the logistic regression model is $\mathrm{pi}^{\wedge} \wedge 2 / 3$. The overdispersion and underdispersion were checked using the generalized linear model quasibinomial family.

A 2-explanatory-variable logistic regression model-a quantitative one (nCCD index) and a binary one (TTT, $\leq 3$ hours versus $>3$ hours) - with interaction was selected using a Bayesian information criterion stepwise analysis to estimate the probability (Y) of full MCA recanalization at 24 hours.

Considering $\mathrm{X} 1=\mathrm{nCCD}$ and $\mathrm{X} 2=\mathrm{TTT} \pm 3$ hours, the graph of $\mathrm{Y}$, function of $\mathrm{nCCD}$, in patients treated within 3 hours from stroke onset was calculated as follows:

$\mathrm{P}[\mathrm{Y}=1 \mid \mathrm{X} 1=\mathrm{x} 1, \mathrm{X} 2=\mathrm{x} 2]=$

$\mathrm{F}\left(\right.$ beta_0 + beta_ $1^{\star} \mathrm{x} 1+$ beta_ $2^{\star} \mathrm{x} 2+$ beta_ $\left.3^{\star} \mathrm{x} \_1^{\star} \mathrm{x} \_2\right)$,

where $\mathrm{x} 2=0$ (corresponding to a TTT $\leq 3$ hours) or $\mathrm{x} 2=1$ (corresponding to a TTT $>3$ hours) and $\mathrm{x} 1$ (nCCD) varies over 


\begin{tabular}{|c|c|c|c|c|c|}
\hline & Age & $\begin{array}{l}\text { Sex Ratio } \\
\text { (M/F) }\end{array}$ & $\begin{array}{l}\text { Diabetes } \\
(\%)\end{array}$ & $\begin{array}{l}\text { Antiplatelet } \\
\text { Therapy (\%) }\end{array}$ & $\begin{array}{c}\text { Stroke Etiology } \\
\text { (CE/Atherom/Other) (\%) }\end{array}$ \\
\hline \multicolumn{6}{|l|}{ Recanalizers + Nonrecanalizers } \\
\hline Distal MCA-M1 occlusion $(n=23)$ & $67.5(15.1)$ & $7 / 16$ & 0 & 26 & $60.8 / 8.7 / 30.4$ \\
\hline Proximal MCA-M1 occlusion ( $n=26$ ) & 63 (16.7) & $8 / 18$ & 11.5 & 34.6 & $69.2 / 3.8 / 26.9$ \\
\hline ICA-MCA M1 occlusion $(n=15)$ & $66(10.2)$ & $14 / 1$ & 0 & 33 & $00 / 93.3 / 6.6$ \\
\hline All $(n=64)$ & $65(14.8)$ & $30 / 34$ & 4.7 & 31.2 & $50 / 26.5 / 23.5$ \\
\hline \multicolumn{6}{|l|}{ Recanalizers } \\
\hline Distal MCA-M1 occlusion $(n=19)$ & 66 (16.2) & $7 / 12$ & 0 & 21 & $57.9 / 10.5 / 31.5$ \\
\hline Proximal MCA-M1 occlusion $(n=16)$ & $59.8(20)$ & $6 / 10$ & 12.5 & 43.7 & $68.7 / 00 / 31.2$ \\
\hline ICA-MCA Ml occlusion $(n=11)$ & $66.7(10.2)$ & $11 / 0$ & 0 & 36.3 & $00 / 100 / 00$ \\
\hline All $(n=46)$ & $62.5(18)$ & $13 / 33$ & 4 & 22 & $46 / 28 / 24$ \\
\hline \multicolumn{6}{|l|}{ Nonrecanalizers } \\
\hline Distal MCA-M1 occlusion $(n=4)$ & $74.7(4.6)$ & $1 / 3$ & 0 & 50 & $75 / 00 / 25$ \\
\hline Proximal MCA-M1 occlusion $(n=10)$ & $68(8.4)$ & $2 / 8$ & 10 & 20 & $70 / 10 / 20$ \\
\hline ICA-MCA Ml occlusion $(n=4)$ & $64(11.5)$ & $3 / 1$ & 0 & 25 & $00 / 75 / 25$ \\
\hline All $(n=18)$ & $69(8.88)$ & $6 / 12$ & 6 & 28 & $56 / 22 / 22$ \\
\hline
\end{tabular}

Note:-Recanalizers = arterial occlusive lesion (AOL) 3 recanalization, ie, full recanalization; nonrecanalizers $=\mathrm{AOL} 0-2$ recanalization, ie, partial or null recanalization. Univariate statistical analysis comparing recanalizers with nonrecanalizers. Results are expressed as mean (standard deviation), ratio, or percentages. CE/Atherom/Other indicates cardioembolic, atheromatous, and other etiology.

Table 4: Baseline characteristics, nCCD values, and outcome in recanalizers and nonrecanalizers

\begin{tabular}{|c|c|c|c|c|c|}
\hline & TTT (min) & $\begin{array}{l}\text { NIHSS } \\
\text { Score }\end{array}$ & $\begin{array}{l}\text { DWI Vol } \\
\text { (ml) }\end{array}$ & $\begin{array}{c}\text { Collateral Circulation } \\
\text { Deficit (nCCD) }\end{array}$ & $\begin{array}{c}\% \text { mRS } 0-2 / 6 \\
\text { at } 3 \text { Months }\end{array}$ \\
\hline \multicolumn{6}{|l|}{ Recanalizers + Nonrecanalizers } \\
\hline Distal MCA-M1 occlusion ( $n=23$ ) & $181.5(91.2)$ & $8.6(5.9)$ & $13.9(16.4)$ & $73.3(53.12)$ & $78.2 / 4.3$ \\
\hline Proximal MCA-M1 occlusion $(n=26)$ & $175.4(58.4)$ & $12(5.7)$ & $16.7(14)$ & $92.1(51.3)$ & $54 / 3.8$ \\
\hline ICA-MCA M1 occlusion $(n=15)$ & $158.86(65.6)$ & $9.86(5.15)$ & $20.77(14)$ & $104.1(77.3)$ & $60 / 6.6$ \\
\hline All $(n=64)$ & $174(72.7)$ & $10(5.8)$ & $17(14.9)$ & $88.17(59.21)$ & $64 / 4.7$ \\
\hline \multicolumn{6}{|l|}{ Recanalizers } \\
\hline Distal MCA-M1 occlusion ( $n=19,82.6 \%$ ) & $164.4(88.7)$ & $8(5.8)$ & $12.1(12.2)$ & $68.2(42.3)$ & $89.5 / 0$ \\
\hline Proximal MCA-M1 occlusion ( $n=16,61.5 \%$ ) & $158.4(46.1)$ & $11.4(5.4)$ & $13.8(11.5)$ & $92.7(47.3)$ & $68.7 / 0$ \\
\hline ICA-MCA M1 occlusion ( $n=11,73.3 \%)$ & $155(71.2)$ & $9.27(5.31)$ & $17.6(11.1)$ & $86.6(69.2)$ & $63.6 / 9.1$ \\
\hline All $(n=46,71.8 \%)$ & $157.5(68.2)$ & $9.3(5.7)$ & $13(12)$ & $79.6(46.4)$ & $76 / 2$ \\
\hline \multicolumn{6}{|l|}{ Nonrecanalizers } \\
\hline Distal MCA-M1 occlusion ( $n=4,17.4 \%$ ) & $262.5(56.4)$ & $11.5(6.1)$ & $22.3(30.8)$ & $97.85(96.7)$ & $25 / 25$ \\
\hline Proximal MCA-M1 occlusion ( $n=10,38.5 \%$ ) & $202.7(67.8)$ & $13.1(6.3)$ & $21.4(17)$ & $91(59.7)$ & $30 / 10$ \\
\hline ICA-MCA Ml occlusion ( $n=4,26.7 \%)$ & $172(53.4)$ & $11.5(5)$ & $29.5(19)$ & $152(88.2)$ & $50 / 0$ \\
\hline All $(n=18,28.1 \%)$ & $209.1(67.2)^{\mathrm{a}}$ & $12.4(5.7)^{\mathrm{a}}$ & $23.4(19.9)^{a}$ & $106.1(74.1)$ & $33^{a} / 11$ \\
\hline
\end{tabular}

Note:-Recanalizers = arterial occlusive lesion (AOL) 3 recanalization, ie, full recanalization; nonrecanalizers $=A O L 0-2$ recanalization, ie, partial or null recanalization. Univariate statistical analysis comparing recanalizers versus nonrecanalizers. Student $t$ test or Fisher exact test for comparison of percentages. Results are expressed as mean (standard deviation), ratio, or percentages. mRS indicates modified Rankin Scale; DWI Vol, volume of lesions detected on DWI.

${ }^{\mathrm{a}} \mathrm{P}<0.05$.

the observed values spectrum within the sample, and the beta_i coefficient values were estimated by the maximum likelihood method.

The statistical significance threshold was set at $P<.05$.

\section{RESULTS}

Of the total sample, $47 \%$ of the patients were male and $4.7 \%$ had diabetes. The mean age was $64 \pm 14.8$ years. The mean baseline NIHSS score was $10 \pm 5.8$, mean TTT was $174 \pm 72.7$ minutes; 23.4\% had a combined ICA and MCA occlusion, but patients with $\mathrm{T}$ occlusion were not included in this study because they were preferentially given endovascular treatment.

In the whole series, the etiology of stroke was cardioembolic in $50 \%$ of the cases and atheromatous in $26.5 \%$. The etiology of MCA occlusion was atheroembolic in most cases of ICA-MCA occlusion (embolus migration from an acute occlusion of an atheromatous proximal tight ICA stenosis). In cases of isolated MCA occlusion, patients with atheroembolic etiology had multiple vascular risk factors and ICA atheromatosis without significant stenosis and/or aortic atheromatosis, without other etiology detected. Only 1 isolated MCA occlusion was related to intracranial atheromatosis, possibly because of the small number of diabetic patients in this series.

Twenty-four hours after IV thrombolysis, MCA-M1 recanalization was complete (arterial occlusive lesion 3 ) in $71.9 \%$ of the cases, partial (arterial occlusive lesion 1-2) in 18.7\%, and null (arterial occlusive lesion 0) in 9.4\% (arterial occlusive lesion scoring $\left.{ }^{17}\right)$. At 3 months, $64 \%$ of the patients had a modified Rankin Scale of $0-2$ (good outcome). Death occurred in 4.7\%. More details on the patient population are presented in Tables 3 and 4 .

The small cohort of 7 patients exceptionally treated with IV thrombolysis between 4.5 and 5.5 hours had full MCA recanalization (arterial occlusive lesion 3) at 24 hours in 57\% of the cases. No hemorrhagic transformation occurred, and, 3 months after stroke onset, the modified Rankin Scale score was 0 in $57 \%$ of the cases and 3 in $43 \%$. 


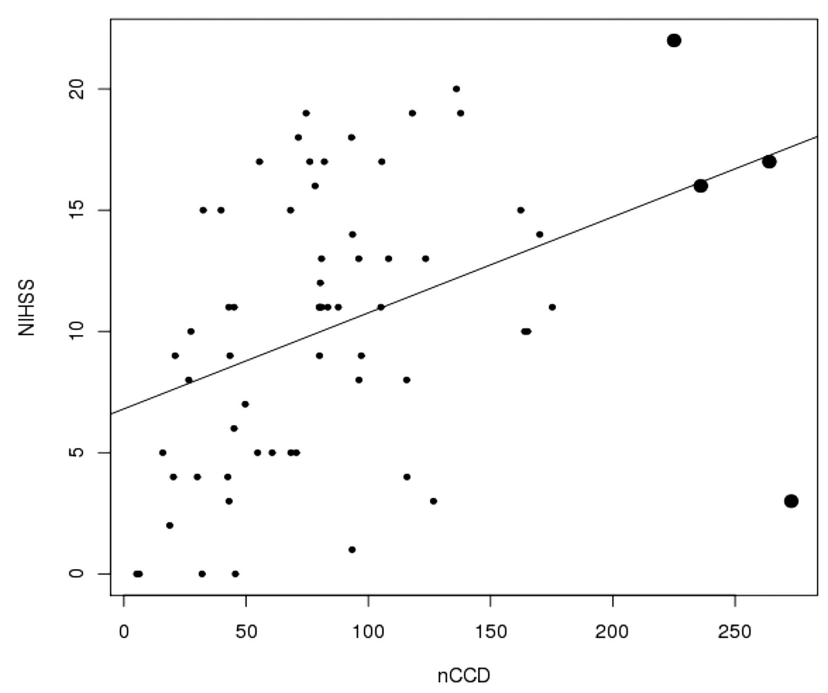

FIG 2. Values dispersion illustrating the significant correlation between nCCD and baseline NIHSS. The bold dots represent the outliers (Pearson correlation test, positive estimated correlation $=0.40, P=$ .00089)

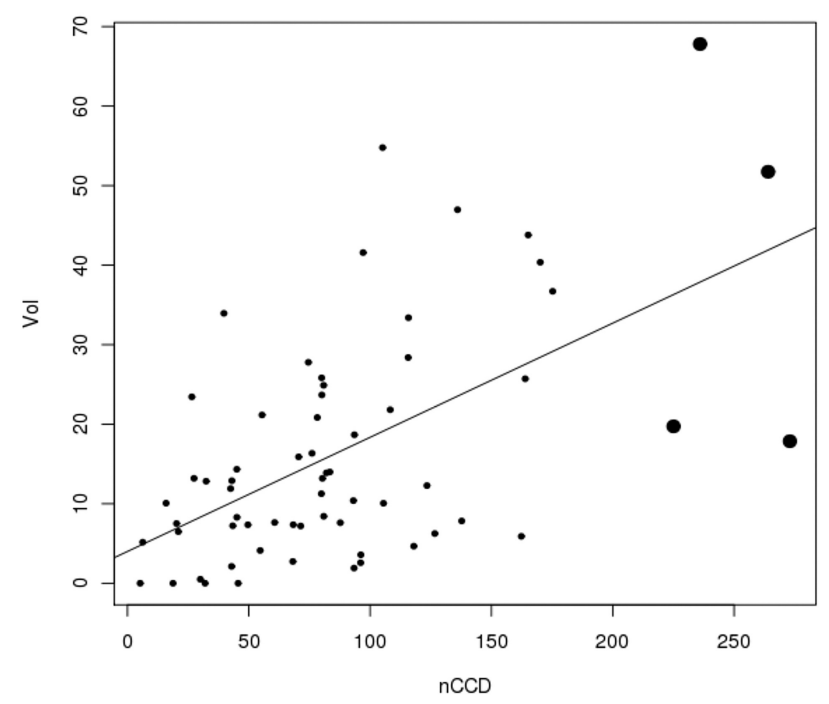

FIG 3. Values dispersion illustrating the significant correlation between $\mathrm{nCCD}$ and lesional volume on DWI. The bold dots represent the outliers (Pearson correlation test, positive estimated correlation $=0.57, P<.0001$.

\section{Statistical Analysis Results}

Compared with collateral circulation deficit, the normalization of collateral circulation deficit with regard to the contralateral Tmax mean value (nCCD) reduced the values dispersion and outliers. It also homogenized the population (significant decrease of variance, unilateral $F$-test, $P=.004$ ).

The nCCD is significantly correlated with NIHSS (Fig 2) and with the lesional volume in DWI (Fig 3) (Pearson correlation test, $P=.00089$ and $P<.0001)$. The higher the nCCD, the higher the NIHSS score and lesional volume will be (positive estimated correlations, respectively equal to 0.40 and 0.57 ).

The nCCD was not significantly correlated with the TTT in the overall population (Pearson test, $P=.58$ ).

There was no statistically significant difference of nCCD based on the type of arterial occlusion (ANOVA Fisher test, $P=.27$ ).
When the entire study population was considered, absent any adjustment upon TTT, no significant correlation was found between the nCCD and the MCA-M1 recanalization at 24 hours (correlation ratio test, eta2 $=0.036$ and $P=.13$ ).

However, patients who did not recanalize were treated significantly later than the fully recanalized ones (Table 4 , Student $t$ test, $P=.0069$ ).

Moreover, after adjustment by the degree of clinical severity (NIHSS), the TTT still remains a predictor for recanalization at 24 hours in the study population $(P=.029$, Wald test; odds ratio 0.99 ; $95 \%$ CI $0.982-0.999)$. Thus, when considering the adjustment by NIHSS, a 1-hour increase of the TTT increases the odds of not observing full recanalization at 24 hours by 1.77 times, whereas the odds are 5.56 times higher for a 3-hour TTT increase.

Considering that the recanalizers and nonrecanalizers were not adjusted upon the TTT, we investigated the potential influence of collateral flow on the MCA-M1 recanalization at 24 hours, taking into account the TTT.

Within the 0 - to 1.5 -hour time window $(n=8)$, all patients were fully recanalized, preventing any correlation analysis of nCCD influence on MCA recanalization.

Within the time windows of $1.5-3$ hours $(n=29), 0-3$ hours $(n=37)$, and $0-4.5$ hours $(n=57)$, the nCCD is significantly correlated with full MCA recanalization at 24 hours (respective correlation ratio tests and statistical significance: eta2 $=0.2614$, $P=.00459$; eta2 $=0.23, P=.0023$; eta $2=0.068, P=.0495)$. However, the wider time window ( $0-4.5$ hours) is associated with a much lower correlation ratio test value compared with shorter time windows, and the corresponding $P$ value is very close to the statistical significance threshold.

In addition, when considering the dichotomized time windows of TTT less or more than 3 hours, the nCCD is significantly correlated with complete MCA recanalization within the 3-hour time-window only (correlation ratio test, eta $2=0.23, P=.0023$, before 3 hours; eta $2=0.0020, P=.85$, within the 3 - to 4.5 -hour time window; eta $2=0.0033, P=.77$, within the 3 - to 5.5 -hour time window).

Furthermore, among those treated within 3 hours (Fig 4), patients who did not achieve full recanalization showed significantly higher nCCD than fully recanalized ones (Mann-Whitney $U$ test, $P=.007)$.

After a stepwise analysis selection (using Bayesian information criterion; value $=71.9$ ) of the determining parameters among the explanatory variables - nCCD, TTT (coded 0/1 for before/after 3 hours), antiplatelet therapy, etiology, site of arterial occlusion, and their second-order interaction-the only 3 parameters to take into account in the prediction of full MCA-M1 recanalization are the nCCD, the TTT ( $\leq 3$ hours/ $>3$ hours), and their interaction.

The logistic regression analysis considering the nCCD, the TTT ( \pm 3 hours), and their interaction showed that the NCCD is significantly predictive of full recanalization at 24 hours when the TTT is $\leq 3$ hours (odds ratio $0.97,95 \%$ CI $0.97-0.99$; Wald test, $P=.021)$.

Furthermore, the probability of recanalization significantly decreases with the increase of nCCD within the first 3 hours after stroke onset $(P=.021)$ (Fig 5). Thus, for patients treated within 


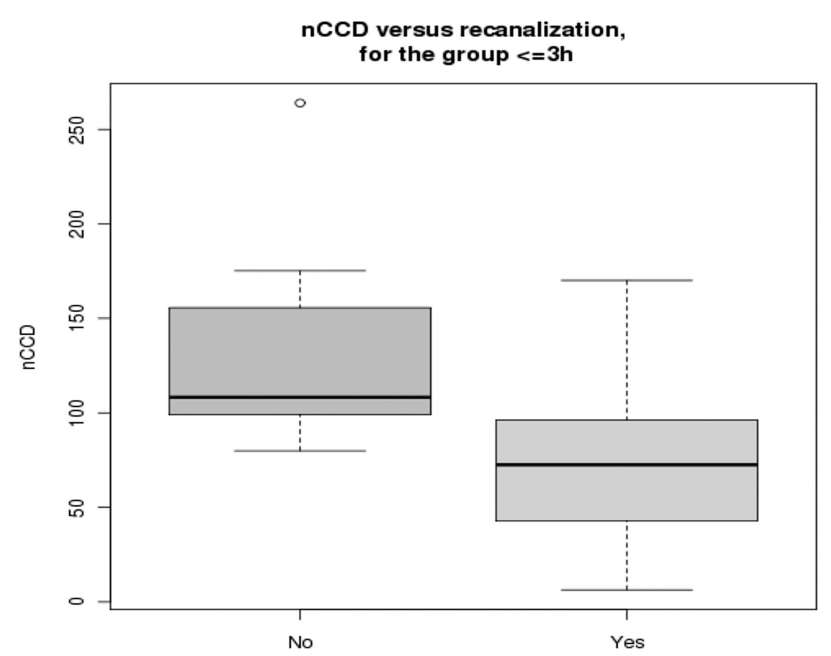

FIG 4. $\mathrm{nCCD}$ values in patients treated within 3 hours from stroke onset, function of the recanalization status (partial or null versus full). The $\mathrm{nCCD}$ is significantly higher in patients who did not achieve full recanalization $(P=.007)$.

the first 3 hours, the probability of achieving full recanalization decreases by 0.033 for a nCCD increase of 10 units around the mean value (mean nCCD $=88.16$ ). Similarly, when nCCD increases from 50 to 150 , the probability of full recanalization decreases from 0.94 to 0.53 .

After 3 hours, the probability of full recanalization tends to slightly increase with the increase of nCCD. However, the increase of this probability is not statistically significant $(P=.762)$.

In addition, the univariate analysis showed that the nCCD is significantly and inversely correlated to the recovery of independence at 3 months after stroke onset (correlation ratio test, eta $2=$ $.0941, P=.0137$; simple linear regression model considering the nCCD and the binary independence variable, modified Rankin Scale $\leq 2$ versus $>2$, significant beta $=-37.56$ ).

The logistic regression analysis considering the nCCD, age, TTT(minutes), and full recanalization at 24 hours showed that the nCCD remains a predictor for independent recovery at 3 months after stroke onset, similar to the full recanalization at 24 hours (Hosmer-Lemeshow test, $P=.81$; $\mathrm{nCCD}=$ odds ratio 0.988, 95\% CI 0.977-0.999, Wald test, $P=.042$; full MCA-M1 recanalization at 24 hours $=$ odds ratio $4.539,95 \%$ CI $1.252-$ 16.456, Wald test, $P=.021$ ).

\section{DISCUSSION}

In patients with MCA occlusion treated with IV thrombolysis, Liebeskind ${ }^{1}$ suggested that a good CC may augment recanalization by delivering the drug to the distal end of the clot (retrograde collateral filling).

To test this hypothesis, we checked whether the quality of CC, which was indirectly evaluated using MR-PWI, impacted the full MCA-M1 recanalization rate. This recanalization rate was assessed 24 hours after IV thrombolysis using a 64-detector CT scanner, a good noninvasive alternative to angiography. ${ }^{18}$

Full MCA recanalization within 24 hours post-IV thrombolysis is a strong predictor, per se, of good stroke outcome at 3 months. ${ }^{19}$ That is not surprising, as arterial patency evaluated 24 hours after IV thrombolysis results from either early full and per-

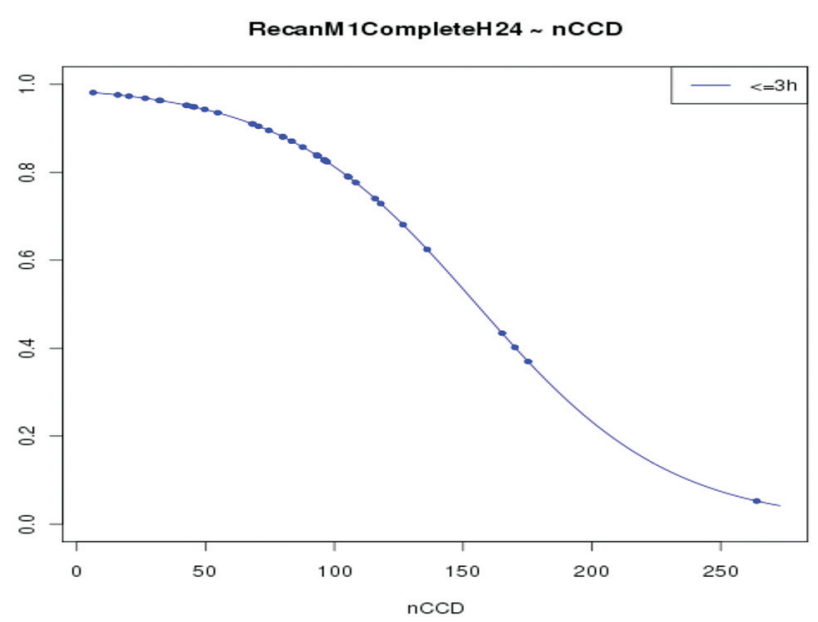

FIG 5. Probability of full MCA-M1 recanalization at 24 hours, function of nCCD value in patients treated within 3 hours after stroke onset. The probability of recanalization significantly decreases with the increase of $\operatorname{nCCD}(P=.021)$.

manent recanalization, which is another independent predictor of good outcome at 3 months, ${ }^{20}$ or slow progressive recanalization or reocclusion that occurs in $34 \%$ of patients with any early post-IV thrombolysis recanalization. ${ }^{21}$ Thus, full MCA recanalization at 24 hours is an important criterion that must be taken into consideration to assess the benefit of IV thrombolysis and the impact of any factor that might interfere with its efficiency, such as collateral flow.

In the current study, we evaluated the quality of CC with a new collateral flow index (nCCD), which was derived from Tmax maps. Tmax, the time-to-maximum of the flow-scaled residue function, represents a perfusion characteristic related to the flow of the contrast agent bolus from the selected feeding artery to the respective tissue site. ${ }^{22}$ Furthermore, Liebeskind ${ }^{1}$ suggested that gradation of abnormality intensity on time parameter maps (like Tmax) may be a specific marker for CC in case of acute ischemia. nCCD, calculated from Tmax maps, is an index of this gradation. In addition, the nCCD index allows a much more precise measure than a 3 -grade collateral score based on CTA. ${ }^{3}$ Indeed, this technique might overestimate the strength of the CC because of the little information provided by CTA about flow itself. ${ }^{3}$ This information, provided by PWI, is paramount because patients with residual flow signals detected by transcranial Doppler before IV tPA bolus are twice as likely to have early complete recanalization. ${ }^{23}$

\section{Correlation Analysis between nCCD and Baseline NIHSS, Volume of Baseline DWI Abnormalities, and 3-Month Outcome}

The nCCD index is significantly and positively correlated with baseline NIHSS values and volume of acute ischemic lesions detected on DWI. Moreover, multiple logistic regression analysis identified baseline nCCD and full MCA-M1 recanalization at 24 hours as independent predictors of functional independence at 3 months.

These results strengthen the link between nCCD and CC. Indeed, as expected for an index of collateral flow deficit, the higher 
the nCCD value, the higher the baseline NIHSS score, the higher the lesional volume, and the worse the outcome. ${ }^{1}$

The highly reproducible measure of the nCCD index makes it potentially useful to noninvasively evaluate future strategies aiming to improve collateral flow. ${ }^{24}$

\section{Influence of Time-to-Treatment on Post-IV Thrombolysis Recanalization Rate}

In the current series, patients who did not reach complete recanalization were treated significantly later compared with fully recanalized ones (Table 4). After adjustment for baseline stroke severity, the TTT was still a significant predictor of full MCA-M1 recanalization at 24 hours. To our knowledge, most studies in the literature did not report time dependency of full MCA-M1 recanalization 24 hours after IV thrombolysis, ${ }^{19,25}$ probably because they only dealt with patients treated within the 3-hour time window, whereas the current series is a post-European Cooperative Acute Stroke Study III ${ }^{9}$ series, with $42 \%$ of the patients treated beyond 3 hours after stroke onset. It is well known that the longer a clot persists over time, the denser and more resistant to fibrinolysis it becomes, until a hard clot-for example, a fibrin-attenuated clot-is formed. ${ }^{26}$ Interestingly, Röther et $\mathrm{al}^{27}$ also demonstrated that the time point of tPA therapy significantly affects the recanalization rate in subgroups of patients treated with IV thrombolysis within 6 hours and dichotomized by the 3-hour limit, just as described in the current series.

\section{Predictive Value of nCCD on Post-IV Thrombolysis Recanalization Rate}

Taking into account the significant influence of the TTT on the recanalization rate, correlation analysis between nCCD and recanalization was performed in patients of the series dichotomized by the 3-hour limit. This analysis showed that baseline nCCD is significantly correlated with recanalization only within the 3-hour time window. Moreover, as expected, patients who did not achieve complete recanalization have a significantly higher nCCD compared with fully recanalized patients. The analysis also showed that the probablility of full MCA-M1 recanalization significantly decreases as the nCCD increases.

Besides the confirmation of the initial hypothesis suggesting a possible link between CC and post-IV thrombolysis recanalization, ${ }^{1}$ these data also suggest that, beyond 3 hours, CC potentiates the action of IV tPA on the clot with less efficiency. However, considering the relatively low number of patients who did not achieve full recanalization, no definitive conclusions about correlations between nCCD and recanalization in patients treated with IV thrombolysis beyond 3 hours can be drawn here. This hypothesis does not cast any doubt over the ability of intra-arterial thrombolysis (higher in situ concentration of the drug injected in the thrombus) or thrombectomy (mechanical effect) to recanalize MCA occlusion beyond 3 hours. Nor does it call into question the fact that a good collateral flow might facilitate recanalization beyond 3 hours during intra-arterial thrombolysis, thanks to a potential lower clot burden due to a reduced intravascular stagnant flow. ${ }^{28}$

\section{Limitations of the Study}

The first limitation of the study is the lack of investigation into the correlation of nCCD index value and angiographic collateral circulation grading, which is the reference standard technique for this purpose. This invasive method cannot be applied to patients treated with IV thrombolysis alone. However, this correlation could be easily investigated in patients who underwent MR-PWI before endovascular therapy for acute cerebral ischemia. ${ }^{6}$ Regardless, the nCCD index is a new predictor of full MCA-M1 recanalization and stroke outcome after IV thrombolysis, easily available through a noninvasive technique.

The second limitation is the retrospective design of the study. However, patients analyzed in this series have been included in a prospective clinical registry from a single stroke center with highly homogeneous and standardized medical care, with 3-month follow-up completed for all patients.

The third limitation is the comparison of MRA and CTA in diagnosing MCA recanalization. Indeed, time-of-flight MRA may overestimate the degree of initial MCA occlusion and, thereby, the ability of IV thrombolysis to fully recanalize complete MCA-M1 occlusion. However, this bias was minimized, as MRA was systematically followed by transcranial Doppler monitoring, confirming the full MCA-M1 occlusion. ${ }^{29}$

The fourth limitation is the very small number of diabetic patients. Indeed, the frequency of post-IV thrombolysis vessel reopening is markedly reduced in subjects with diabetes. ${ }^{19}$ Therefore, the results of this study should not be extrapolated to diabetic patients.

The last limitation is the confounding effect of prolonged transcranial Doppler performed in all patients of this series during IV thrombolysis. The positive effect of transcranial Doppler on MCA recanalization has been suggested by several authors and must be taken into account in the analysis of the current data and conclusions. ${ }^{30}$ As a result, the relatively high rate of MCA recanalization observed in the current study could be partially explained by the small number of diabetic patients and the use of prolonged transcranial Doppler during IV thrombolysis. Therefore, the synergistic effect of collateral flow on clot dissolution induced by tPA could be lower in diabetic patients treated with IV thrombolysis without coupled prolonged transcranial Doppler.

\section{CONCLUSIONS}

The current study demonstrates how MR perfusion-weighted imaging can provide an index of collateral flow that is predictive of the rate of recanalization of MCA-M1 occlusions in patients treated with IV thrombolysis. This index, nCCD, is based on Tmax maps at different time points and is highly reproducible. This new metric may provide useful information for better patient selection for therapy and for developing strategies aimed to augment collateral flow. However, further studies are necessary to fully confirm our findings.

\section{ACKNOWLEDGMENTS}

As the head of the stroke center of La Timone University Hospital (Marseille, France) during this study, Prof. F. Nicoli managed all patients of the current series and thanks all nurses in his department for their devotion to their patients. 
Disclosures: Francois Nicoli-UNRELATED: Consultancy: Olea Medical; Stock/Stock Options: Minor share holder in Olea Medical. Pierre Lafaye de Micheaux-RELATED: Consulting Fee or Honorarium: Olea Medical, Comments: Statistical Analysis. Nadine Girard-UNRELATED: Consultancy: Olea Medical, Comments: The money is not paid to me but for travel and accommodations for scientific meetings.

\section{REFERENCES}

1. Liebeskind DS. Collaterals in acute stroke: beyond the clot. Neuroimag Clin N Am 2005;15:553-73

2. Angermaier A, Langner S, Kirsch M, et al. CT-angiographic collateralization predicts final infarct volume after intra-arterial thrombolysis for acute anterior circulation ischemic stroke. Cerebrovasc Dis 2011;31:177-84

3. Lima FO, Furie KL, Silva GS, et al. The pattern of leptomeningeal collaterals on CT angiography is a strong predictor of long-term functional outcome in stroke patients with large vessel intracranial occlusion. Stroke 2010;41:2316-22

4. Nogueira RG, Liebeskind DS, Sung G, et al. Predictors of good clinical outcomes, mortality and successful revascularization in patients with acute ischemic stroke undergoing thrombectomy: pooled analysis of the mechanical embolus removal in cerebral ischemia (MERCI) and multi MERCI trials. Stroke 2009;40:3777-83

5. Tan IYL, Demchuk AM, Hopyan J, et al. CT angiography clot burden score and collateral score: correlation with clinical and radiologic outcomes in acute middle cerebral artery infarct. AJNR Am J Neuroradiol 2009;30:525-31

6. Bang OY, Saver JL, Buck BH, et al. Impact of collateral flow on tissue fate in acute ischaemic stroke. J Neurol Neurosurg Psychiatry 2008; 79:625-29

7. McVerry F, Liebeskind DS, Muir KW. Systematic review of methods for assessing lepto-meningeal collateral flow. AJNR Am J Neuroradiol 2012;33:576-82

8. Tissue plasminogen activator for acute ischemic stroke. The National Institute of Neurological Disorders and Stroke rt-PA Stroke Study Group. N Engl J Med 1995;333:1581-87

9. Hacke W, Kaste M, Bluhmki E, et al. Thrombolysis with alteplase 3 to 4.5 hours after acute ischemic stroke. N Engl J Med 2008;359: 1317-29

10. Nicoli F, Squarcioni C, Grimaud L, et al. Results of intravenous thrombolysis using MR imaging selection: a series of 420 acute ischemic stroke patients. 2010 World Stroke Conference, Seoul. Int J Stroke 2010;5:99

11. Mouridsen K, Christensen S, Gyldensted L, et al. Automatic selection of arterial input function using cluster analysis. Magn Reson Med 2006;55:524-31

12. Wu O, Østergaard L, Weisskoff RM, et al. Tracer arrival timinginsensitive technique for estimating flow in MR perfusionweighted imaging using singular value decomposition with a blockcirculant deconvolution matrix. Magn Reson Med 2003;50:164-74

13. Takasawa M, Jones PS, Guadagno JV, et al. How reliable is perfusion MR in acute stroke? Validation and determination of the penumbra threshold against quantitative PET. Stroke 2008;39:870-77
14. Olivot JM, Mlynash M, Thijs VN, et al. Optimal Tmax threshold for predicting penumbral tissue in acute stroke. Stroke 2009;40:469-75

15. R Development Core Team. R: A language and environment for statistical computing, $\mathrm{R}$ Foundation for Statistical Computing version 2.11.0 (2010-04-22). Vienna, Austria: http://www.R-project.org

16. Long JS. Regression Models for Categorical and Limited Dependent Variables. Thousand Oaks, CA: Sage Publications, 1997

17. Khatri P, Neff J, Broderick JP, et al. Revascularization end points in stroke interventional trials: recanalization versus reperfusion in IMS-I. Stroke 2005;36:2400-03

18. Han A, Yoon DY, Chang SK, et al. Accuracy of CT angiography in the assessment of the circle of Willis: comparaison of volume-rendered images and digital substraction angiography. Acta Radiol 2011;52: 889-93

19. Zangerle A, Kiechl S, Spiegel M, et al. Recanalization after thrombolysis in stroke patients: predictors and prognostic implications. Neurology 2007;68:39-44

20. Molina CA, Alexandrov AV, Demchuk AM, et al. Improving the predictive accuracy of recanalization on stroke outcome in patients treated with tPA. Stroke 2004;35:151-57

21. Alexandrov AV, Grotta JC. Arterial reocclusion in stroke patients treated with intravenous tPA. Neurology 2002;59:862-67

22. Fieselmann A, Kowarschik M, Ganguly A, et al. Deconvolutionbased CT and MR brain perfusion measurement: theoretical model revisited and practical implementation details. Int J Biomed Imaging 2011 Aug 28 [Epub ahead of print]

23. Saqqur M, Tsivgoulis G, Molina CA, et al. Residual flow at the site of intracranial occlusion on transcranial Doppler predicts response to intravenous thrombolysis: a multi-center study. Cerebrovasc Dis 2009,27:5-12

24. Shuaib A, Butcher K, Mohammad AA, et al. Collateral blood vessels in acute ischaemic stroke: a potential therapeutic target. Lancet Neurol 2011;10:909-21

25. Wunderlich MT, Goertler M, Postert T, et al. Recanalization after intravenous thrombolysis: does a recanalization time window exist? Neurology 2007;68:1364-68

26. Kirchhof K, Sikinger M, Welzel T, et al. Does the result of thrombolysis with recombinant tPA in rabbits depend on the erythrocyteand fibrin-content of a thrombus? Fortschr Röntgenstr 2004;176:98105

27. Röther J, Schellinger PD, Gass A, et al. Effect of intravenous thrombolysis on MRI parameters and functional outcome in acute stroke <6 hours. Stroke 2002;33:2438-45

28. Jovin TG, Gupta R, Horowitz MB, et al. Pretreatment ipsilateral regional cortical blood flow influences vessel recanalization in intra-arterial thrombolysis for MCA occlusion. AJNR Am J Neuroradiol 2007;28:164-67

29. Aoki J, Kimura K, Iguchi Y, et al. A combined TCD and MRA screening for significant siphon portion of internal carotid artery (S-ICA) stenosis. J Neuroimaging 2012;22:172-76

30. Saqqur M, Tsigovlis G, Nicoli F, et al. The role of sonolysis, sonothrombolysis with and without microbubble in acute ischemic stroke: a systematic review and meta-analysis of randomized control trials and case control studies. Stroke 2011;42;e261 